\title{
Polarization-locked temporal vector solitons in a fiber laser: experiment
}

\author{
B. C. Collings* \\ Department of Electrical Engineering, Princeton University, Princeton, New Jersey 08544 \\ and Bell Laboratories, Lucent Technologies, Holmdel, New Jersey 07733
}

S. T. Cundiff

JILA, University of Colorado and National Institute of Standards and Technology, Boulder, Colorado 80309-0440

\section{N. N. Akhmediev}

Australian Photonics Cooperative Research Centre, Optical Sciences Centre, The Australian National University, Canberra, ACT 0200, Australia

\section{J. M. Soto-Crespo}

Instituto de Óptica, Consejo Superior de Investigaciones Cientificas, Serrano 121, 28006 Madrid, Spain

\section{K. Bergman}

Department of Electrical Engineering, Princeton University, Princeton, New Jersey 08544

\author{
W. H. Knox
}

Bell Laboratories, Lucent Technologies, Holmdel, New Jersey 07733

Received May 3, 1999; revised manuscript received October 18, 1999

\begin{abstract}
We experimentally observe polarization-locked vector solitons in a passively mode-locked fiber laser. The vector soliton pulse is composed of components along both principal polarization axes of the linearly birefringent laser cavity. For certain values of birefringence and pulse energy these components propagate with a constant relative optical phase of $\pm \pi / 2$, and hence the pulse has a fixed elliptical polarization state. The linear birefringence of the cavity is canceled by the nonlinear birefringence created by the unequal amplitudes of the two polarization components. This dynamic equalization of the phase velocities of the components results in the stable propagation of an elliptically polarized vector soliton pulse. Under different conditions we also observe the nonlinear instability of the fast principal axis as an intracavity pulse linearly polarized along the slow axis of the cavity. We present the experimental characterization of both the polarization-locked vector soliton and the fast axis instability and discuss the nonlinear mechanism creating both phenomena. (C) 2000 Optical Society of America [S0740-3224(00)01003-1]
\end{abstract}

OCIS codes: $060.5530,190.4370,140.4050,320.7090,140.3500$.

\section{INTRODUCTION}

The propagation of temporal solitons and solitonlike pulses in nonlinear media has been the subject of considerable theoretical and experimental research. This has resulted in a wealth of interesting physical phenomena and practical applications. ${ }^{1-4}$ Soliton propagation is typically addressed with a scalar theory; i.e., the vector nature of the polarization state is ignored despite the fact that all single-mode fiber (SMF) supports two orthogonal polarization modes. ${ }^{1}$ Furthermore, asymmetries in the fiber waveguide caused during manufacturing, externally applied stress, or bending lead to random birefringence (both magnitude and principal axes orientation) in the fiber. ${ }^{5}$ Thus the polarization state of a (nonsoliton) pulse in this environment evolves as its two components propagate at differing phase velocities. ${ }^{5}$ Unequal group veloci- ties result in a polarization-dependent splitting of the initial pulse. For typical fiber-optic communication systemsthe random evolution of the polarization state with propagation does not impose a significant impairment if polarization-insensitive devices are used; however, pulse splitting resulting from unequal group velocities (polarization-mode dispersion) can cause significant degradation. ${ }^{6}$

A fundamental soliton pulse propagates in fiber without changes in either its pulse shape or its optical spectrum by means of balancing anomalous group velocity dispersion (GVD) with the self-phase modulation (SPM). Optical fiber solitons were first predicted and are typically modeled as solutions to the scalar (linear polarization) nonlinear Schrödinger equation. ${ }^{3}$ However, for a complete treatment of pulse propagation in standard 
(non-polarization-maintaining) fiber, the differences in the propagation velocities of both polarization modes as well as nonlinear coupling mechanisms must be included. Menyuk described the vector nature of nonlinear pulse propagation in birefringent fiber with two coupled nonlinear Schrödinger equations (CNLSE's). ${ }^{7}$ Theoretical investigations have shown that a stable pulse composed of both polarization components can propagate as a single vector soliton unit, overcoming their group velocity difference. $^{7-9}$ This effect has been experimentally observed. ${ }^{10}$ The general term vector soliton refers to a multidimensional entity that propagates in an invariant or periodic manner in an otherwise destructive environment. $^{11}$ A temporal pulse composed of orthogonally polarized components with equalized group velocities is an example of a vector soliton. Since the two components of this vector soliton pulse have a nonlinearly induced common group velocity, we refer to this type of vector soliton as a group-velocity-locked vector soliton. Since the components of a group-velocity-locked vector soliton do not necessarily have a common phase velocity, its total polarization state evolves according to its birefringent environment. In a randomly birefringent fiber the polarization state evolution is correspondingly random. Evangelides et al. showed that, for this case, the Stokes vector of the pulse's polarization state, on average, covers the entire Poincaré sphere during the random evolution. $^{12}$ Furthermore, it has been shown that the group-velocity-locked vector soliton is well approximated by a linearly polarized soliton pulse provided that the nonlinear refractive-index coefficient is properly modified. ${ }^{7,12}$

For low-birefringence conditions in which the linear birefringence is comparable with potential nonlinear birefringence, the group velocity difference is assumed to be negligible. Within this regime elliptically polarized vector soliton solutions of the CNLSE's have been found that maintain both their temporal and polarization state profiles during propagation within a birefringent environment. ${ }^{13-18}$ These stationary, single solitons solutions are composed of two orthogonally polarized and optically phase-locked components and form a class of vector solitons called phase- or polarization-locked vector solitons (PLVS). For these solutions the relative phase between the components is locked at $\pm \pi / 2$, but the polarization state profile across the pulse is not uniform. However, that profile is invariant with propagation despite the birefringent environment in contrast to the group-velocity-locked vector soliton. The elliptical polarization state of the pulse in conjunction with the SPM, cross-phase modulation (XPM), and the coherent energy coupling (CEC, also known as four-wave mixing) creates a nonlinear birefringence that dynamically balances the linear birefringence. This balance, similar to that between the GVD and SPM of a fundamental soliton, enables the PLVS pulse to propagate without changes to its polarization state.

In this paper we describe in detail the experimental observation and characterization of a PLVS in an opticalfiber laser cavity. ${ }^{19}$ Under the proper conditions we observe the formation of a PLVS pulse that propagates without change of its polarization state within a linearly birefringent, passively mode-locked fiber laser cavity. The optical phase between the orthogonally polarized components along the principal birefringent axes of the cavity is measured to be fixed at $\pm \pi / 2$. The difference between the intensities of the components, the nonlinear birefringence, depends directly on the magnitude of linear birefringence. These results are in qualitative agreement with numerical solutions for the PLVS. ${ }^{20}$ The dependence of the spectral widths of the two components of the PLVS on birefringence and pulse-energy-dependent behavior is also shown to be in qualitative agreement with the numerical PLVS solutions.

The PLVS pulses are solutions of the conservative CNLSE's, whereas the laser is not a conservative system. ${ }^{14-16}$ However, the magnitude of the perturbations to the pulse from the elements of the laser cavity are relatively small in this laser. ${ }^{21}$ A companion paper investigates this difference through numerical simulation of the entire laser system described here, including the significant nonconservative effects and a direct comparison with an analogous conservative system. ${ }^{20}$ These results indicate that the solutions to this nonconservative laser system have much in common with the PLVS pulse solutions of a conservative, lossless birefringent fiber. ${ }^{14-18,20}$ Both conservative and nonconservative results are compared with the experimental measurements and are described within the companion paper. ${ }^{20}$

The PLVS solutions have been found to be weakly unstable. ${ }^{15-17}$ Therefore, for the indefinite propagation of the PLVS pulse within the laser cavity, this fragile balance between the linear and the nonlinear birefringence requires a stabilizing mechanism able to suppress perturbative effects. Numerical analysis shows that the contribution of the XPM and the CEC provides the negative feedback mechanism necessary to prevent the growth of random perturbations. ${ }^{15,17,20}$ Furthermore, the damping of perturbations is believed to be the result of the nonconservative effects of the laser and mode-locking mechanism and is addressed by the companion paper. ${ }^{20}$

For different regions of laser cavity birefringence we observe a second effect: the nonlinearity-induced instability of the polarization state of light linearly polarized along the fast axis of linearly birefringent fiber. This effect has been theoretically predicted for both $\mathrm{cw}^{22}$ and soliton propagation. ${ }^{23}$ This instability arises when the magnitudes of the linear and the nonlinear birefringences are comparable and destabilizes the polarization state of a pulse polarized along the fast axis. Conversely, a polarization state along the slow axis is stable. This effect is clearly manifested in our fiber laser as a pulse with a linear polarization state locked along the slow axis of the cavity. Instability of the fast axis has been observed in a single-pass transmission experiment in which the polarization state of a soliton evolved away from the fast axis. $^{24}$ In contrast to a single-pass experiment, the pulse in the laser effectively propagates an infinite distance, allowing the instability to fully mature.

Here we present the experimental observation of PLVS pulses. Before presenting the details, we briefly outline the theoretical background, which is also fully developed in Ref. 20. Then we describe our experiment and measurement techniques, present the characterization of the 
PLVS pulse, and qualitatively compare these findings with the predictions of the numerical solutions. We discuss the important stabilizing mechanism formed by the $\mathrm{XPM}$ and the CEC effects, which suppress perturbations to the PLVS. We then present the experimental results illustrating the instability of the fast axis and discuss the parameters that govern the domination of the instability.

\section{THEORETICAL BACKGROUND}

The full vector model describing the propagation of two components orthogonally polarized along the principal axes of a lossless fiber is given by the conservative CNLSE's ${ }^{7}$ :

$$
\begin{aligned}
i \frac{\partial u}{\partial z}+i \delta \frac{\partial u}{\partial t}+\gamma u & +\frac{1}{2} \frac{\partial^{2} u}{\partial t^{2}} \\
& +\left(|u|^{2}+A|v|^{2}\right) u+B v^{2} u^{*}=0, \\
i \frac{\partial v}{\partial z}-i \delta \frac{\partial v}{\partial t}-\gamma v & +\frac{1}{2} \frac{\partial^{2} v}{\partial t^{2}} \\
& +\left(|v|^{2}+A|u|^{2}\right) v+B u^{2} v^{*}=0,
\end{aligned}
$$

where $u$ and $v$ are the component envelopes along the slow and the fast axes, respectively; $t$ and $z$ are the normalized time and distance; $2 \delta$ and $2 \gamma$ are the normalized group and phase velocity differences, and $A$ and $B$ are the XPM and the CEC coefficients, respectively.

The linearly polarized fundamental soliton solutions include than $1 \mathrm{~W}$. For this case, solutions of the CNLSE's correspond to two pulses, orthogonally polarized along the birefringent axes, which mutually trap each other and propagate as a single nondispersing vector soliton unit. $^{8-10}$ The XPM coupling causes the central optical frequencies of one component to increase and the other to decrease. In conjunction with a frequency-dependent group velocity these shifts equalize their group velocities. ${ }^{7}$ In the absence of the nonlinearity the components of a pulse will retain their common central frequency and travel at unequal group velocities resulting in the temporal splitting of the two components. Owing to the large phase-velocity difference between components, the polarization state of this vector soliton pulse evolves rapidly with propagation. Nevertheless, at any given point, the same polarization state applies to the entire pulse, since the two components have the same shape and phase profile. ${ }^{12}$ Standard soliton communications systems operate in this high-birefringence regime and use group-velocity-locked vector soliton pulses. Hence these systems are relatively immune to the detrimental pulsesplitting effects of random and unequal group velocities (polarization-mode dispersion), which often impair the performance of linear (nonsoliton) communication systems. ${ }^{6,27}$

In the low-birefringence case the linear birefringence is comparable with the possible nonlinear birefringence (because it depends on the polarization state), and the difference between the group velocities is typically negligible and ignored. Furthermore, since the relative optical

$\begin{array}{ll}u(\tau, z)=U_{0} \operatorname{sech}(\tau-\delta z) \exp (i \gamma z), & v(\tau, z)=0, \\ u(\tau, z)=0, & v(\tau, z)=V_{0} \operatorname{sech}(\tau+\delta z) \exp (i \gamma z) .\end{array}$

For an isotropic and conservative medium, $B=1$ - $A$. When $A=1$ (the SPM and the XPM coefficients are equal, and CEC vanishes) and $\delta=0$, the CNLSE's are integrable with stationary phase-locked solutions. ${ }^{25}$ The experimental observation of these simple hyperbolicsecant-shaped solutions, known as Manakov solitons, is difficult, because $A \neq 1$ for most low-loss materials. Through engineering of the SPM and the XPM coefficients in an anisotropic waveguide $(A \sim 0.95)$, spatial Manakov solitons (also governed by analogous CNLSE's with the same solutions) have been observed. ${ }^{26}$ In isotropic media such as standard SMF, $A=2 / 3$ and rigorous temporal Manakov solitons cannot occur.

When the linear birefringence is significantly larger than the nonlinear birefringence, the relative optical phase between components varies so rapidly that all phase-dependent effects effectively average to zero with propagation. ${ }^{7}$ Thus the CEC and the phase velocity difference terms of the CNLSE's can be ignored, resulting in only incoherent XPM coupling between the two components. Solutions to this case approximate Manakov solitons, especially for elliptically birefringent fiber where $A$ can be engineered to be unity. ${ }^{7}$ Within this paper this condition is referred to as high birefringence and occurs, for example, in SMF with pulses of peak powers of less phase, phase velocity difference, and CEC terms must be retained in the CNLSE's, the components are both incoherently and coherently coupled. For this case theoretical analysis has found three lowest-order (nodeless) stationary solutions of the CNLSE's: the two fundamental soliton solutions linearly polarized along either the fast or the slow axes given by Eqs. (2) and (3) and a (numerical) solution with an elliptical polarization. ${ }^{14-16}$ This elliptically polarized soliton is a PLVS, since it contains energy in both components and propagates without change of its polarization state. The components have a relative phase of $\pm \pi / 2$ but are not necessarily of the same functional shape; thus the polarization state is not uniform across the pulse. ${ }^{14-16}$ Other stationary solutions of the CNLSE found to date are of higher order ${ }^{11,13,16}$ and have at least one node in one or both components. In contrast to solitons linearly polarized along the fast axis whose polarization state has been found to be unstable ${ }^{23}$ the elliptically polarized soliton solutions possess a weak, oscillatory instability. ${ }^{14-17}$

For PLVS propagation the phase velocities of the two components must be equal so that the polarization state remains unchanged. This is analogous to the requirement of equal group velocities for a group-velocity-locked vector soliton to avoid polarization-dependent splitting. 
As mentioned above, the nonlinear birefringence is created by an unequal distribution of energy between components and compensates the linear birefringence. This balance effectively equalizes the phase velocities of the components. Equivalently, because of the linear birefringence, the refractive index of the slow axis is greater than that of the fast axis. With an asymmetric intensity distribution between axes, the nonlinear contribution to the indices is correspondingly asymmetric. If the larger intensity component is along the fast axis, the nonlinear contribution to the index of the fast axis is greater than that of the slow axis thereby reducing the difference between the total indices. The balance occurs when the difference between the nonlinear index contributions is equal and opposite to the index difference created by the linear birefringence.

The nonlinear birefringence depends on the difference between the intensities of the components or, equivalently, the ellipticity of the polarization state. Thus, with the proper balance, the ellipticity of its polarization state depends directly on the linear birefringence, $\gamma$. When the profiles of both components are approximated to be equal, this relationship can be quantized and is found to be linear and given by

$$
|V|^{2}-|U|^{2}=(\gamma / g)
$$

where $U$ and $V$ are the time-integrated amplitudes of $u$ and $v, g \approx 4 / 3 q(1-A)$, and $q$ is a soliton parameter that is inversely proportional to the soliton period and proportional to the pulse energy squared. ${ }^{14,15}$ Equation (4) also illustrates that $|V|^{2} \geqslant|U|^{2}$ with the component along the fast axis possessing the greater intensity. The magnitude of the nonlinear birefringence of a pulse is limited by its energy and width (implicitly here through normalization) such that a PLVS cannot exist for a linear birefringence of $\gamma>\left(|V|^{2}+|U|^{2}\right) g$. Equivalently, this limit occurs as $|U|^{2} \rightarrow 0$, and the polarization state approaches linear polarization along the fast axis.

\section{EXPERIMENT}

The fiber laser (described in Ref. 21) and the experimental setup are shown in Fig. 1. The laser consists of two lengths of standard SMF and a 17-cm piece of (single spatial mode) $\mathrm{Er} / \mathrm{Yb}$ fiber providing gain. ${ }^{21}$ The round-trip cavity is approximately $430-\mathrm{cm}$ in length, which corresponds to a $48-\mathrm{MHz}$ repetition rate. The mode locking is started and stabilized by a semiconductor passive saturable absorber known as a saturable Bragg reflector. ${ }^{28}$ The saturable Bragg reflector is butt coupled to one end of the linear cavity acting as a saturable high-reflecting mirror. The solitonlike pulse shaping occurring within the cavity dominates the characteristics of the steady-state

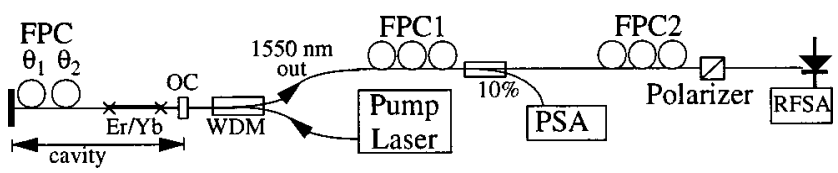

Fig. 1. Schematic of the fiber laser cavity and output diagnostics. FPC, fiber polarization controller; PSA, polarization-state analyzer; WDM, wavelength division multiplexer; RFSA, radiofrequency spectrum analyzer; OC, output coupler.
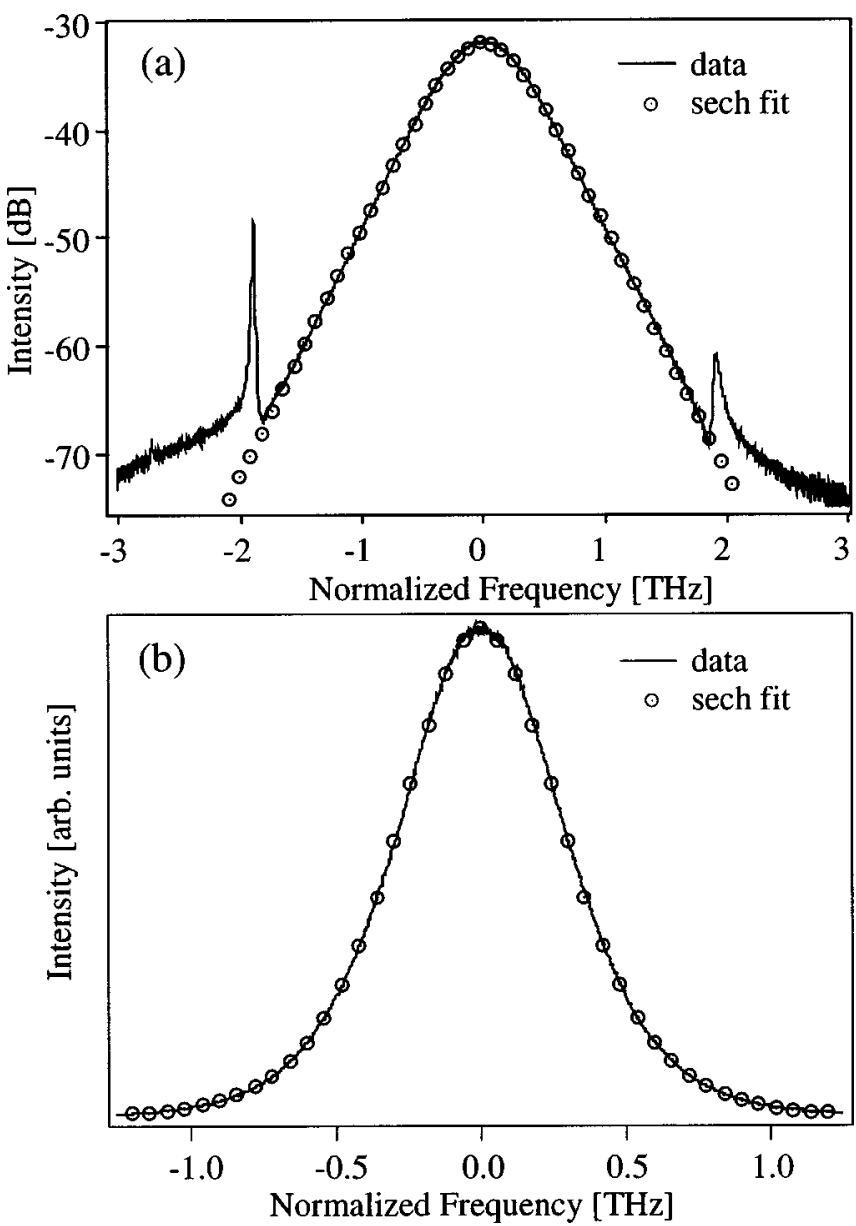

Fig. 2. Polarization-independent optical spectrum of the PLVS (curve) and $\operatorname{sech}^{2}(\nu)$ function fit (circles) versus frequency, $\nu$, plotted on both $\log (\mathrm{a})$ and linear (b) scales.

pulse. $^{21}$ The average GVD of the cavity, consisting of 396 $\mathrm{cm}$ of SMF $(+17 \mathrm{ps} / \mathrm{nm} / \mathrm{km})$ and $34 \mathrm{~cm}$ of $\mathrm{Er} / \mathrm{Yb}(-9.5 \mathrm{ps} /$ $\mathrm{nm} / \mathrm{km}$ ), is $+14.5 \mathrm{ps} / \mathrm{nm} / \mathrm{km}$ (anomalous). Mode locking produces stable $350-800-\mathrm{fs}$ pulses (full-width at halfmaximum) for pulse energies (controlled by variation of the pump power) of $160-65 \mathrm{pJ}$. We estimate the total saturated gain and loss per round trip to be $<3 \%$. The saturated reflectivity of the saturable Bragg reflector is approximately $99.5 \%$ with $<0.5 \%$ saturation induced modulation of the absorption. We estimate that polarization-state-dependent interactions between the saturable Bragg reflector and pulse are small and do not significantly impact the polarization state of the pulse. When the saturable Bragg reflector is replaced with a standard dielectric high reflector, the laser operates in $\mathrm{cw}$ mode.

The soliton period, assuming a linearly polarized pulse, is given by ${ }^{1}$

$$
z_{0}=\frac{\pi \tau_{0}^{2}}{2\left|\beta_{2}\right|}
$$

where $\tau_{0}$ is the pulsewidth and $\beta_{2}$ is the GVD. For the shortest pulse (350 fs, full-width at half-maximum), $z_{0}$ $=3.5 \mathrm{~m}$, which is roughly equivalent to the round-trip length of the cavity. For the longest pulse, $z_{0} \approx 20 \mathrm{~m}$. Also, the perturbations to the pulse (i.e., from gain, loss, 
GVD) are small. For these reasons the solitonlike pulse does not significantly respond to the local perturbations. ${ }^{29}$ Therefore we approximate this system as a uniform medium with parameters characteristic of the average cavity. ${ }^{20,30}$ This assumption is further supported by the relatively small sidebands in the optical spectrum $(<0.1 \%$ of total energy; see Fig. 2). ${ }^{31,32}$

To allow for adjustment of the linear birefringence of the cavity, a portion of the cavity fiber is wrapped around two 5.5-cm-diameter fiber polarization control paddles with three wraps each (see Fig. 1). For SMF fiber each paddle provides approximately $\pi / 2(\lambda / 4$-wave plate) total linear retardation at $1550 \mathrm{~nm}$ with the fast axis in the bend plane of the fiber. ${ }^{33}$ We measure the azimuthal angles, $\theta_{1}$ and $\theta_{2}$, of the two paddles and the directions of their fast axes relative to an arbitrary constant reference (e.g., the table). The remainder of the cavity is mechanically secured such that the magnitude and the principal axes of the residual birefringence (owing to imperfections in the fiber, bends, strains, etc.) of the remainder of the cavity are also constant. Typically, the residual birefringence is less than $\pi / 4$. Therefore the total cavity birefringence is dominated by the birefringence present in the paddles and is variable from 0 to roughly $2 \pi$ by proper adjustment of $\theta_{1}$ and $\theta_{2}$. Approximately $60 \%$ of the cavity fiber is contained within the fiber polarization controller paddles, and, as stated above, these lengths are considerably shorter than the soliton period of the intracavity pulse. Therefore we characterize the total cavity birefringence by its average. This assumption is experimentally justified below.

\section{CAVITY BIREFRINGENCE MEASUREMENT}

Since the cavity retardance is typically $<2 \pi$ per round trip and the polarization beat length ${ }^{1}$ is comparable with the nonlinear length, the laser is in the low-birefringence regime. For most values of total cavity birefringence, the polarization state of the steady-state intracavity pulse evolves during propagation, owing to the linear birefringence. However, since the pulse does not suffer polarization-dependent splitting from the negligible group velocity difference and is composed of components along both principal axes, it is by definition a groupvelocity-locked vector soliton. The output pulse train then consists of a series of pulses with a polarization state evolving from one pulse to the next. The change in the polarization state between adjacent pulses in the train is the round-trip evolution of the polarization state of the intracavity group-velocity-locked vector soliton pulse.

We characterize the rate of this evolution by passing the output through a linear polarizer and detecting the transmitted intensity with a polarization-insensitive fast photodiode. $^{34}$ A fiber polarization controller is inserted before the polarizer (see Fig. 1) to null any residual birefringence present in the fiber between the output coupler and the linear polarizer so that the polarization state incident on the polarizer coincides with that at the output coupler (see Appendix A). Figure 3 depicts how this polarization-state evolution is mapped into an amplitude modulation of the pulse train by the linear polarizer.

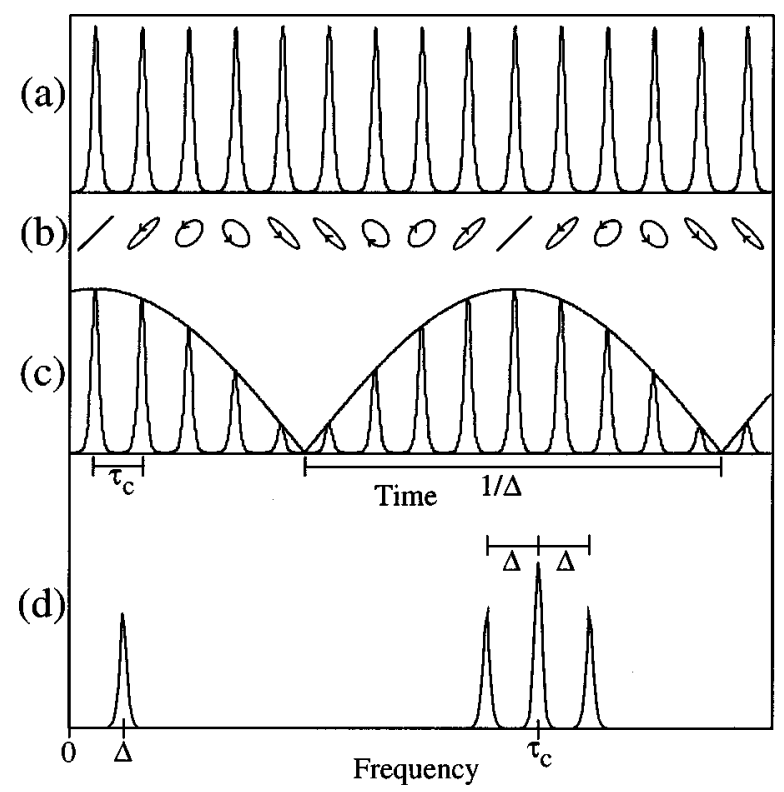

Fig. 3. (a) Schematic of polarization-independent output pulse train of frequency $1 / \tau c$. b) Schematic of the polarization states of the pulses of (a). (c) Schematic of the pulse train of (a) when passed through a linear polarizer aligned along a 45-deg angle or parallel with the polarization state of the leftmost pulse. The period of the intensity modulation of the pulse train is $9 \tau c$ or $1 / \Delta$. (d) Schematic of the frequency spectrum of (c) consisting of a component at the repetition frequency of the pulse train $\left(1 / \tau_{c}\right)$ with sidebands of separation $\Delta$.

To demonstrate this mapping analytically, let $E_{1}(z)$ and $E_{2}(z)$ be the electric fields along the principal birefringent axes of the cavity at the center of the pulse (in normalized units of length) as it propagates through the cavity (neglecting nonlinear effects),

$$
\begin{aligned}
& E_{1}(z)=E_{0} \exp (i \gamma z), \\
& E_{2}(z)=E_{0} \exp (-i \gamma z) .
\end{aligned}
$$

A linear polarizer of azimuthal angle $\theta$ [relative to the azimuthal angle of $\left.E_{1}(z)\right]$ sums the projections of $E_{1}(z)$ and $E_{2}(z)$ along $\theta$. The resulting field is

$$
E(z)=\cos \theta E_{1}(z)+\sin \theta E_{2}(z) .
$$

The photodiode detects the intensity $I(t)$,

$$
\begin{aligned}
I(z)= & |E(z)|^{2}=E_{0}{ }^{2}\left[(\cos \theta)^{2}+(\sin \theta)^{2}\right. \\
& +2 \cos \theta \sin \theta \cos (2 \gamma z)], \\
I(z)= & E_{0}{ }^{2}[1+2 \cos \theta \sin \theta \cos (2 \gamma z)] .
\end{aligned}
$$

Thus the signal current from the photodiode [see Fig. 3(c)] consists of a pulse train with an amplitude modulation of frequency $\Delta=\gamma c / n \pi$, designated the polarization evolution frequency (PEF), where $c$ is the speed of light and $n$ is the refractive index. Monitored with a rf spectrum analyzer, this signal consists of frequency components at the cavity repetition rate and its harmonics, each possessing sidebands with separations of $\Delta$ [see Fig. 3(d)]. The product of the PEF and the round-trip period of the cavity is the number of round trips required for the pulse to undergo a full evolution (a total of $2 \pi$ retardance). Hence the magnitude of the total round-trip cavity birefringence 
is $\beta=2 \pi \Delta \tau_{c}=2 l_{c} \gamma$ (in radians) where $\tau_{c}$ and $l_{c}$ are the round-trip cavity length and period, respectively, which is easily measured by determination of $\Delta$. For a cavity birefringence of larger than $\pi$ the $\mathrm{PEF}$ is aliased below $1 /\left(2 \tau_{c}\right)$, since the polarization state is sampled only once per round trip.

The general structure of the PEF as a function of the birefringence during $\mathrm{cw}$ and mode-locked operation (when the output polarization state is evolving and not locked) is well understood with a simple linear birefringence Jones matrix formalism. ${ }^{34}$ All measurements of the cavity birefringence magnitude are performed during $\mathrm{cw}$ operation to avoid any nonlinear contribution to the evolution.

To determine the azimuthal angles of the principal birefringent axes of the average cavity, we null the magnitude of the PEF components by rotating the angle of the linear polarizer. When the modulation or PEF components vanish, the angle of the polarizer is aligned with a principal axis [e.g., $\theta=0$ or $90 \mathrm{deg}$ in the example of Eq. (9)]. This measurement does not reveal whether the particular axis is fast or slow. The ability to null the PEF components indicates that the nonlinearity of the pulse does not significantly respond to the local birefringence but rather to the average of the total cavity. If the nonlinearity did respond to the local birefringence, or more accurately to the local polarization state, eigenvalues of the system (the principal axis) could not be defined, be- cause of the nonlinear birefringence causing local polarization-state-dependent changes. This justifies averaging the total cavity birefringence as well as treating the pulse as two linearly polarized components along the principal axes of the average cavity.

\section{POLARIZATION-STATE LOCKING}

We observe that, for some values of $\theta_{1}$ and $\theta_{2}$, the polarization-state evolution ceases and all of the pulses in the train possess a common polarization state. ${ }^{34}$ Consequently, the PEF components vanish for all orientations of the polarizer. Figure 4 plots the PEF as a function of $\theta_{1}$ and $\theta_{2}$. The polarization-state locking occurs when the laser is mode locked for nonzero amounts of birefringence; whereas polarization-state evolution occurs for the same amount of birefringence under linear (cw) operation. Since the polarization state of the output pulse train is constant, we measure that state with a polarization-state analyzer. A fiber polarization controller is inserted before the polarization-state analyzer (see Fig. 1) to compensate for the residual birefringence present in the fiber between the output coupler and the polarization-state analyzer such that the polarization state measured by the polarization-state analyzer is consistent with that at the output coupler (see Appendix A).

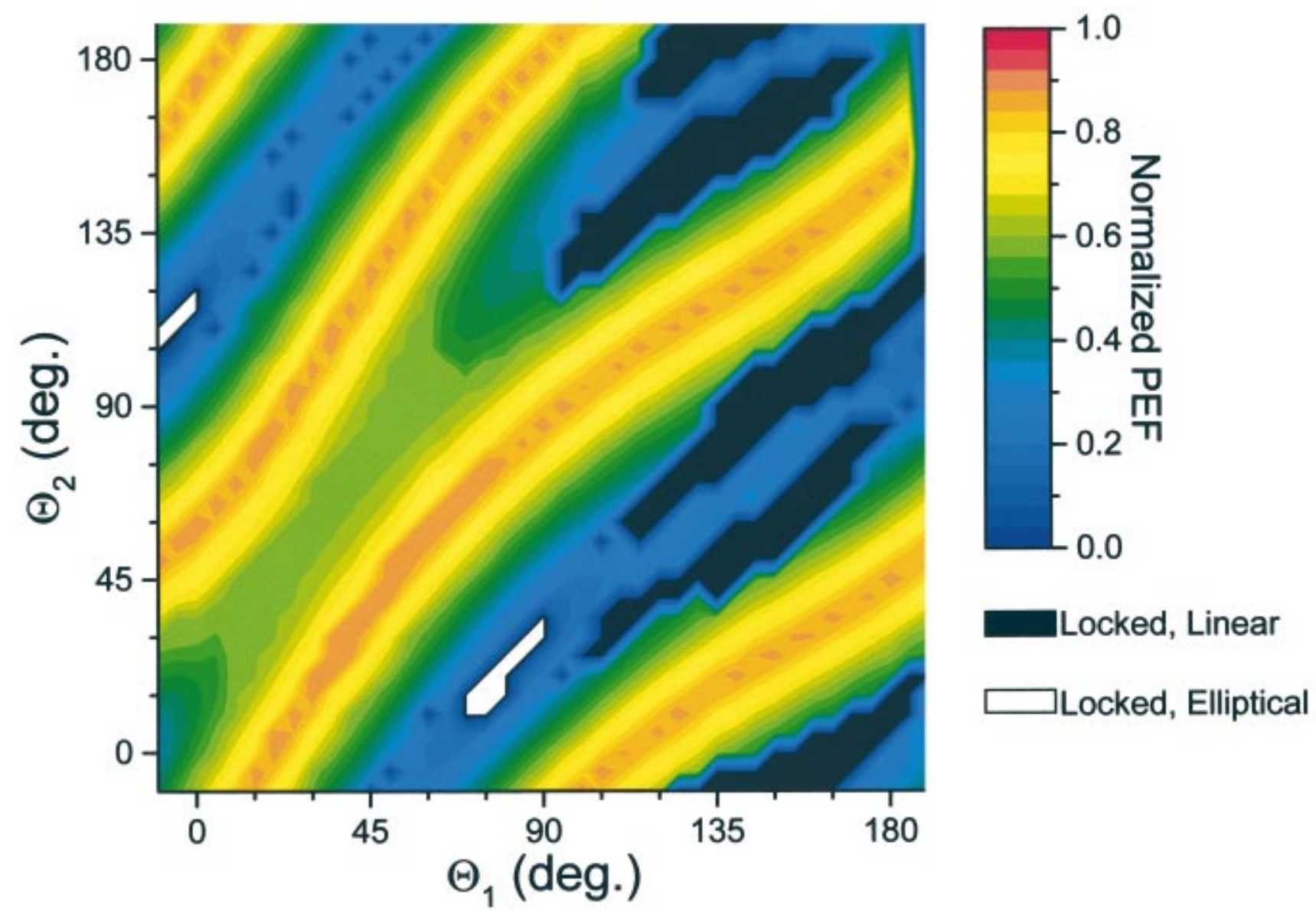

Fig. 4. Plot of PEF versus $\theta_{1}$ and $\theta_{2}$ for (a) high and (b) low pulse energies. The color gradient represents the PEF magnitude (normalized by $1 / 2 \tau_{c}$ ) with black and white indicating regions of locked linearly and elliptically polarized output, respectively. 
We observe two distinct types of locked output, one linearly and one elliptically polarized. In Fig. 4(a) the regions where the linearly and the elliptically polarized locking occur are denoted by black and white, respectively. As is shown below, the elliptically polarized locking corresponds to the formation of a PLVS within the cavity and the linearly polarized locking results from a principal axis instability.

\section{POLARIZATION-LOCKED VECTOR SOLITONS}

To characterize the pulse within the elliptically locked region, we fix $\theta_{1}$ and measure the polarization state of the output with the polarization-state analyzer as a function of $\theta_{2}$ across the elliptically polarized locking region. However, first we replace the saturable Bragg reflector with a high reflector and operate the laser in cw mode. Using a sensitive rf spectrum analyzer and environmental perturbations (i.e., tapping the table), which cause transient mode beating that manifests the PEF, we measure the PEF and hence the birefringence. We also determine the angles of the principal cavity axes as a function of $\theta_{2}$. This measurement reveals that the elliptically polarized locking region is centered about a point of zero birefringence. Estimating the total birefringence from the known birefringence of the individual cavity elements verifies that the total birefringence is indeed zero and not $2 \pi$.

With the laser mode locked, we measure the complete polarization state (all Stokes parameters) of the output as a function of $\theta_{2}$ with the polarization-state analyzer. The elliptically locked output is either left or right handed, depending on the direction (in $\theta_{1}$ and $\theta_{2}$ space) in which the locking region was entered. The amplitude and relative phase of the components along the principal axes are determined by projection of the total measured polarization state onto the previously measured principal axes. The difference between the intensities of the components and their relative phase are plotted in Fig. 5 as a function of the measured total cavity birefringence for both types of elliptical polarization handedness. The linear dependence of this difference on birefringence, characteristic of a PLVS as shown by Eq. (4), is clearly present. Furthermore, the measured relative phase is approximately constant at $+\pi / 2$ or $-\pi / 2$, also characteristic of a PLVS. Finally, the ellipticity and intensity difference is essentially equal for both types of handedness, providing strong evidence that the compensation mechanism is indeed the nonlinear birefringence.

The relative phase between components [see Fig. 5(b)] is approximately constant at either $+\pi / 2$ or $-\pi / 2$ over the entire locking region. The locking is equally stable with both phase values. When the birefringence is varied so that the output polarization becomes unlocked before returning to the locking region, the relative phase can assume either value. For a given birefringence both polarization states with $\pm \pi / 2$ relative phases have the same ellipticity (but opposite handedness) and hence the same intensity difference. Since the intracavity pulse is made up of two components and maintains a fixed relative optical phase and polarization state despite the linear bire- fringence, this intracavity pulse is a PLVS. In contrast, the polarization state of a linear pulse would evolve.

As can be seen in Fig. 5(b), the relative phase deviates from the expected value of $\pm \pi / 2$ at large values of cavity birefringence. We believe this deviation to be an artifact of the location of the output coupler relative to the location of the majority of the "lumped" birefringence at the opposite end of the cavity (see Fig. 1). Since the balance struck between the average linear and nonlinear birefringences, and the nonlinear birefringence is effectively distributed throughout the cavity, local deviation of the relative optical phase away from $\pm \pi / 2$ occurs. Furthermore, this deviation is expected to be greater with increasing total linear birefringence and intensity difference as evident in Fig. 5(b).

The region of cavity birefringence over which the PLVS is observed is power dependent, characteristic of a nonlinear locking mechanism. The intracavity pulse energy is controlled when the pump power is varied. Figure 6 plots the location of the edge of the PLVS locking region versus pulse energy and cavity birefringence. At this edge, the normalized intensity difference is typically within $5 \%$ of unity. This dependence is in agreement with Eq. (4), since higher levels of birefringence cannot be compensated once the normalized intensity difference reaches unity. Thus the maximum birefringence that may be compensated, and for which a PLVS can exist, is dependent on the square of the pulse energy. Experi-
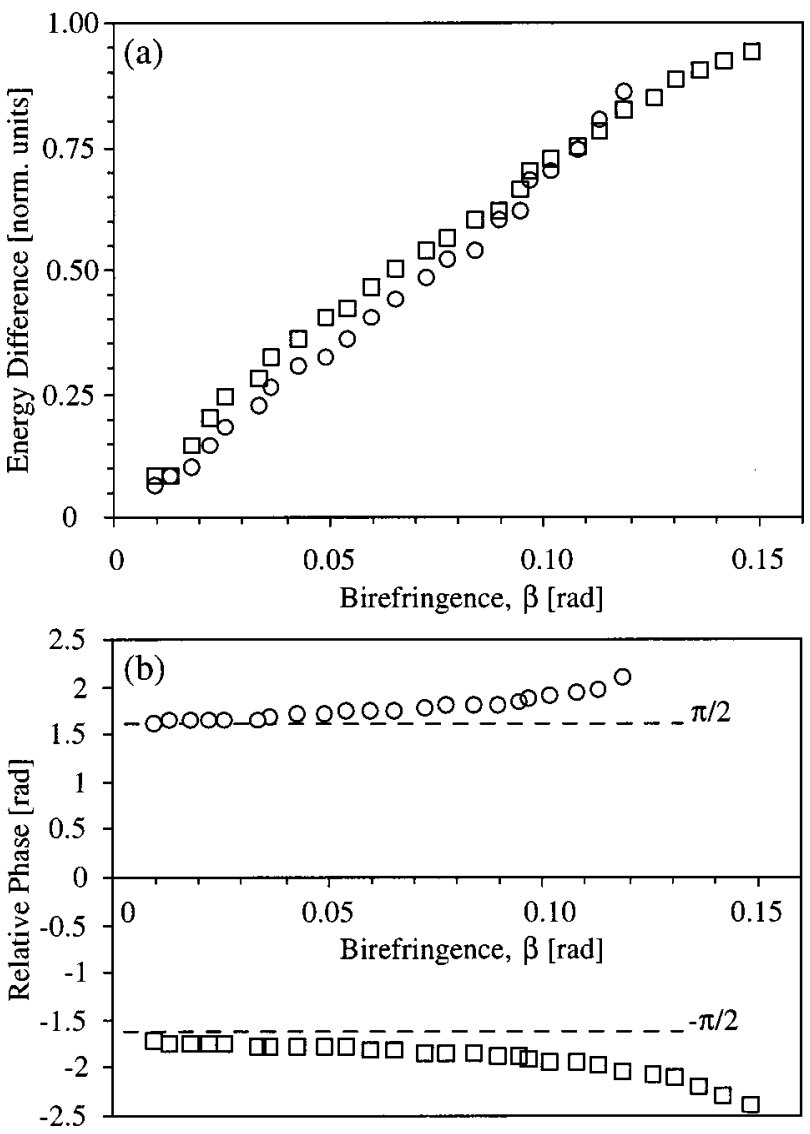

Fig. 5. (a) Intensity difference between the components for both handednesses (normalized by the total intensity) and (b) relative optical phases versus total round-trip cavity retardance, $\beta$. 


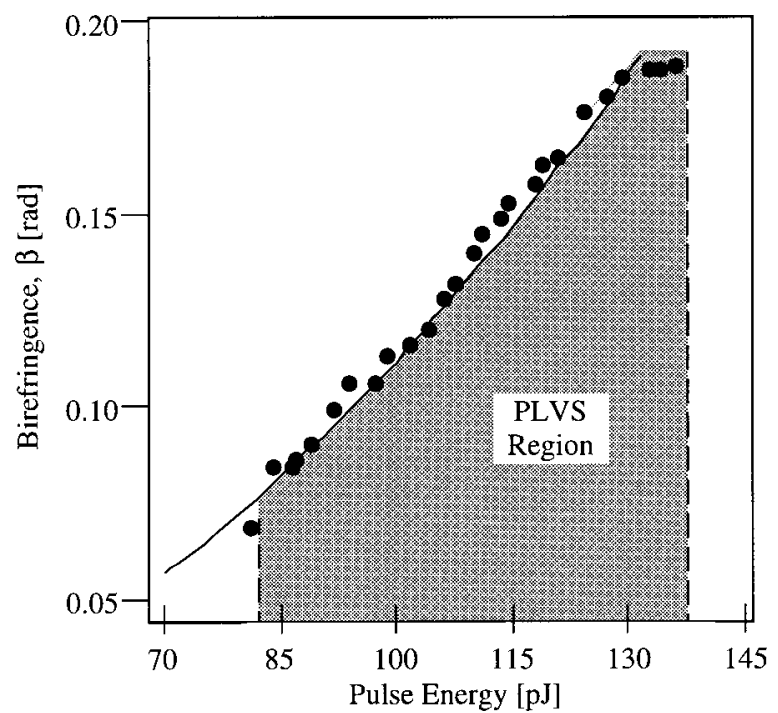

Fig. 6. Maximum round-trip birefringence, $\beta$, where a PLVS is observed versus pulse energy. The solid curve is proportional to the square of the pulse energy.

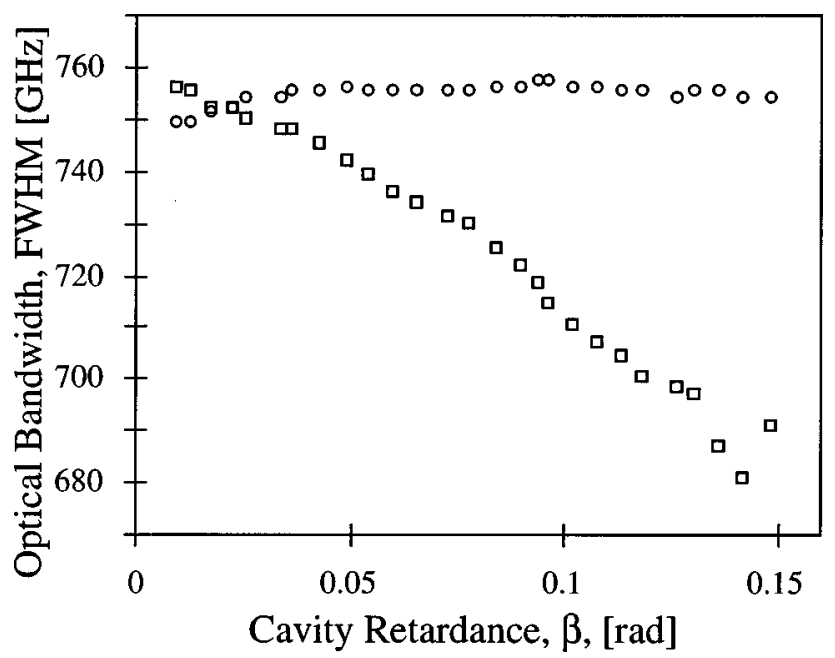

Fig. 7. Measured optical spectral widths of component along the fast axis (circle) and slow axis (square) versus total cavity retardance.

mentally, the location of the edge of the PLVS locking region displays this form of dependence as indicated by the solid curve in Fig. 6.

In Fig. 2 the shape of the (polarization insensitive) intensity optical spectrum as a function of frequency $\nu$, is compared with that of a $\operatorname{sech}^{2}(\nu)$ function with excellent agreement over 4 orders of magnitude. The numerical PLVS solutions of the CNLSE's possess a nonconstant polarization state across the pulse shape as the spectral and corresponding temporal widths of each components diverge with increasing birefringence. ${ }^{14,16}$ For zero birefringence, the polarization state is circular and the two profiles are identical. For increasing birefringence the component along the fast axis of higher intensity becomes short in time and spectrally wider. ${ }^{14,16}$ This behavior is also present in the experimental PLVS. Replacing the photodiode with a polarization-insensitive optical spectrum analyzer allows for measurement of the optical spectra of each component by alignment of the polarizer with a principal axis of the cavity. The measured spectral widths for both components are plotted in Fig. 7. As expected, near zero birefringence, the two components are of comparable widths. With increasing cavity retardance, the component along the fast axis maintains a wider spectral width, also in agreement with theory. ${ }^{14,16,20}$

\section{STABILIZATION MECHANISM}

The boundary of the polarization-locking region is hysteretic. Figure 8 illustrates this hysteresis with a plot of the $\mathrm{PEF}$ ( $\mathrm{PEF}=0$ indicates PLVS) versus both increasing and decreasing $\theta_{1}\left(\theta_{2}\right.$ fixed). Near this boundary the laser is actually tri stable with three possible states of operation: unlocked (polarization evolving) and locked (PLVS) with $\pm \pi / 2$ relative optical phases. This hysteresis suggests the important role that stability plays in the formation of the intracavity PLVS.

The existence of the PLVS depends on the delicate balance of the linear and the nonlinear birefringence as well as the gain and the loss. Its stability requires the suppression of both amplitude and phase perturbations. A change in a component's amplitude translates into a constant drift in the components' relative phase through the unbalanced linear and nonlinear birefringences, resulting in polarization evolution. Likewise, a perturbation to the relative phase causes energy transfer between components through the CEC. This, in turn, results in an amplitude perturbation. Therefore, since the PLVS is stable in the cavity, a mechanism stabilizing the PLVS against perturbations must be present. ${ }^{20}$

The combined action of the CEC, SPM, and XPM provides such a negative feedback mechanism. The CEC transfers energy between components with a direction and magnitude that depend on the relative phase. For a relative phase of $\pm \pi / 2$ and for the proper balance between the nonlinear and the linear birefringences, the net energy transfer is zero. Thus, provided that the amount

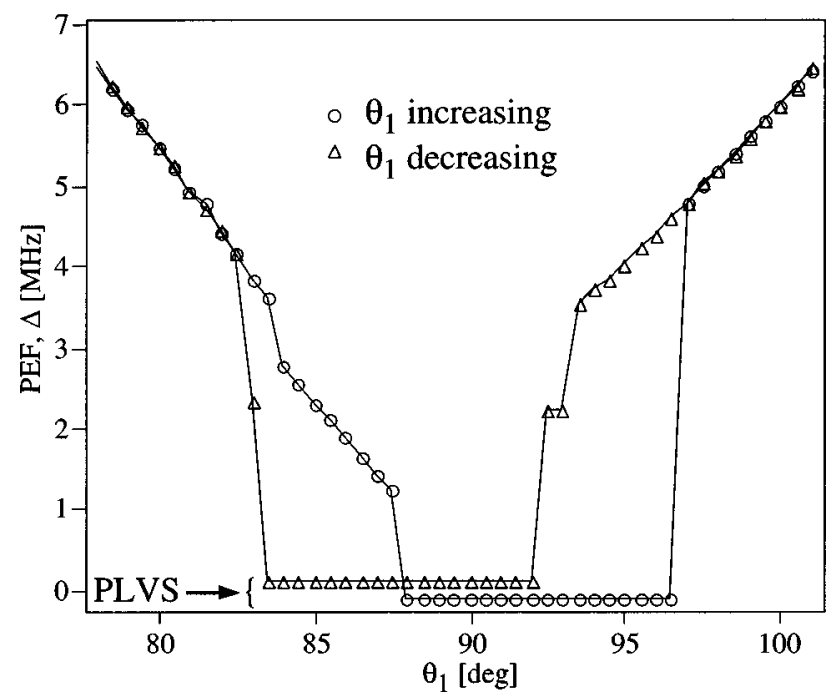

Fig. 8. PEF versus $\theta_{1}$ (circles, $\theta_{1}$ increasing; triangles, $\theta_{1}$ decreasing; $\theta_{2}$ held constant) illustrating hysteresis at the boundary of the PLVS locking region. Elliptical polarization locking (PLVS) is represented as $\mathrm{PEF} \sim 0$ as the data sets are vertically offset in the PLVS region for clarity. 


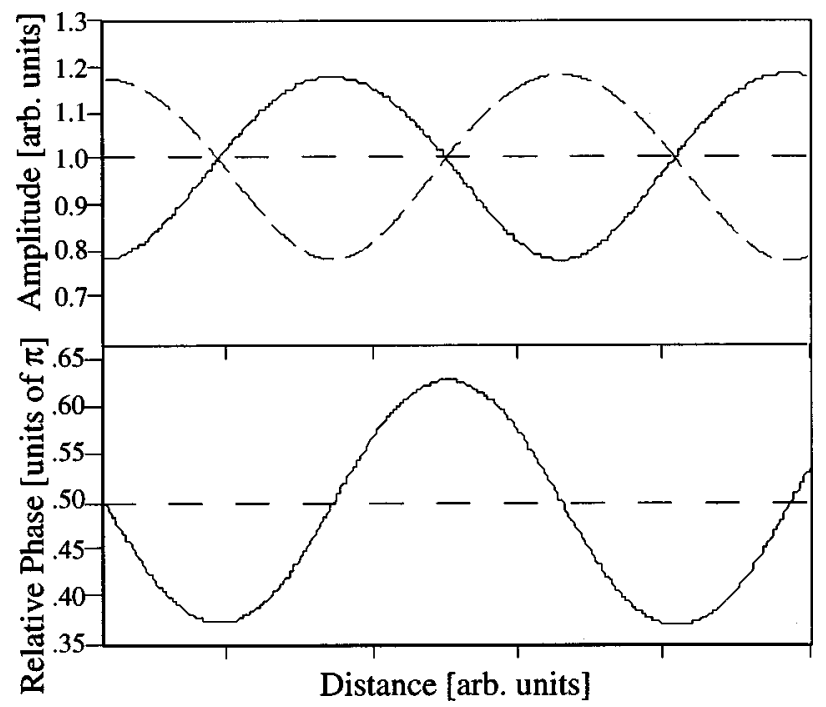

Fig. 9. Schematic of the amplitudes of the two components (dashed and solid curves) and relative phase between components illustrating the interplay and the oscillation between amplitude and relative phase perturbations as a function of propagation distance.

of nonlinear birefringence is correct to compensate the linear birefringence, a relative phase of $\pm \pi / 2$ represents a stationary point, in agreement with the experimentally observed relative phase of the PLVS. Perturbations away from this stable point are corrected by the interaction of the SPM, XPM, and CEC. A relative phase perturbation causes a nonzero net energy transfer by means of the CEC from the component lagging in phase to the component advanced in phase. With a higher intensity, this advanced component suffers a larger nonlinear phase shift than the lagging component (the SPM coefficient is larger than the XPM coefficient). The increased nonlinear phase shift, equivalent to a decrease of the phase velocity of the advanced component, corrects the initial phase perturbation.

An amplitude perturbation causes the component with the increased intensity to lag in phase, owing to its increased nonlinear phase shift. Energy is then transferred through the CEC, as before, to the advanced component, which is the component with the decreased amplitude, and the perturbation is corrected. Thus, these combined actions form a negative feedback system by providing a restoring force toward a relative phase of $\pm \pi / 2$, which balances the nonlinear and the linear birefringences.

This mechanism, however, provides only a restoring force and offers no damping of perturbations. Under these circumstances, initial perturbations oscillate about the central stationary point. The initial disturbance evolves from perturbations in the relative phase to the amplitudes as depicted in Fig. 9. This behavior is analogous to an oscillating pendulum transferring the initial perturbation between displacement and velocity. $\mathrm{Nu}$ merical simulations of this conservative case exhibit this oscillatory behavior. ${ }^{16}$ However, since the experimentally observed PLVS are stationary and stable, perturbations must be damped and dispersed. We conjecture that the nonconservative and dissipative effects of the laser are responsible for the suppression of these oscillations. Numerical simulations of this laser incorporating the saturable gain, loss, gain bandwidth, and saturable absorption show polarization locking with stable, nonoscillatory behavior. ${ }^{20,30}$

\section{FAST AXIS INSTABILITY}

Linear polarization along the fast axis has been shown to become unstable when the nonlinear birefringence is comparable with the linear birefringence (low-birefringence regime). ${ }^{22,26,27}$ For example, with light linearly polarized along the fast axis, the nonlinear refractive-index contribution can increase the total index of the fast axis such that it is comparable with that of the slow axis. Under these circumstances the fiber can become effectively isotropic. However, in an isotropic nonlinear medium, small, random variations in the state of polarization cause further polarization-state evolution (rotation) resulting in the instability of the polarization state of a soliton polarized along the fast axis.

However, if the optical field is linearly polarized along the slow axis, the nonlinearity increases the total index of the slow axis and results in an effectively larger total birefringence. Small, random variations to the linear birefringence are now less likely to significantly alter the total (linear and nonlinear) birefringence of the fiber, and the light continues to propagate linearly polarized along the slow axis.

In our laser the instability of the fast axis is manifested as a locked output polarization, linearly polarized along the slow axis. This locking is depicted as the black regions in Fig. 4. However, the measurement of the cavity principal axes (as described in Appendix A) does not reveal which axis is fast and which is slow. Within the PLVS region the identity of the measured axes is determined, since the higher-intensity component must be along the fast axis for the nonlinear and the linear birefringence to cancel each other. Beginning within the PLVS locking region, $\theta_{1}$ and $\theta_{2}$ are varied along a trajectory toward a linearly polarized locking region while the angles of the fast and the slow axes are tracked. With this method, the fast and the slow axes can be identified for all $\theta_{1}$ and $\theta_{2}$. Figure 10 shows a plot of the angle of the slow axis, the angle of linearly polarized locked output, and birefringence magnitude as a function of $\theta_{2}$ showing that, within the linearly polarized locked regions, the output polarization is aligned with the slow principal axis of the cavity. The linearly polarized output can be nulled by a crossed polarizer to $35 \mathrm{~dB}$, again indicating that the polarization state varies by less than $0.04 \%$ across the pulse shape. Note that this measurement does not determine the degree of linearity of the output polarization state but simply the uniformity of the polarization state across the pulse.

The region of birefringence where the linearly polarized locking occurs is pulse energy dependent as shown in Fig. 11. For a large pulse energy the region is larger and centered about a larger birefringence. As the pulse energy decreases, both the center of the region and its size decreases. This behavior can be understood when we compare the magnitudes of the linear and the nonlinear bire- 


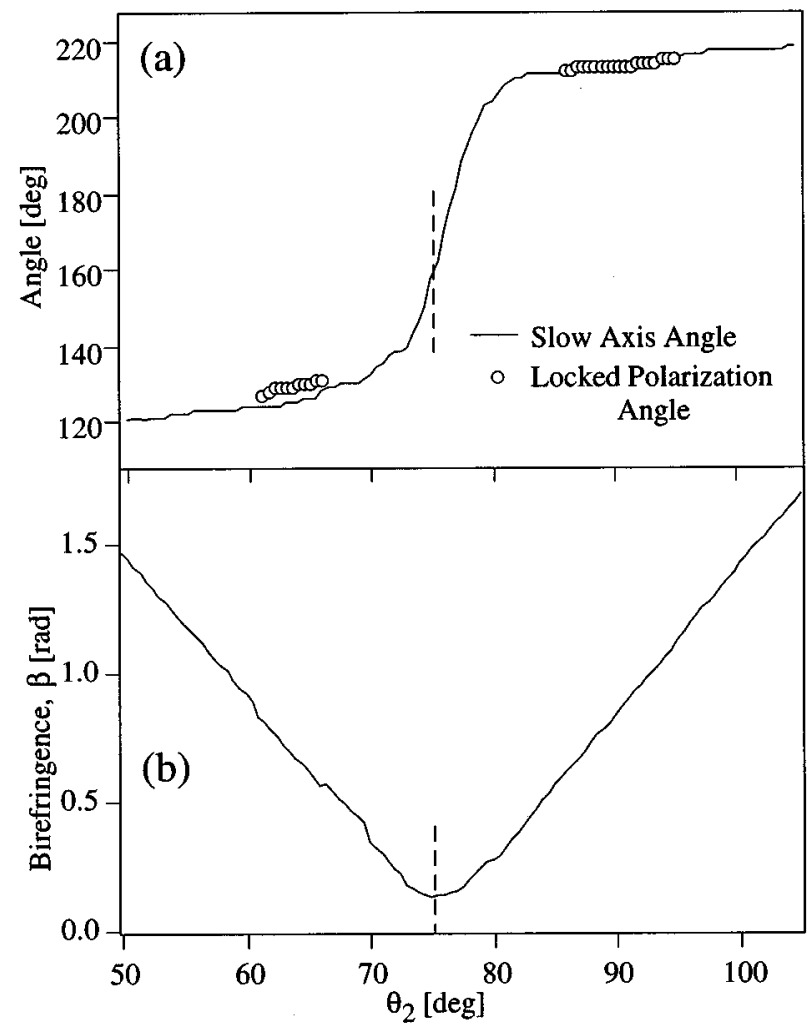

Fig. 10. (a) Measured azimuthal angle of the slow axis (curve) and the linearly polarized locked output (circles). (b) Measured birefringence, $\beta$, versus $\theta_{2}$ ( $\theta_{1}$ held constant).

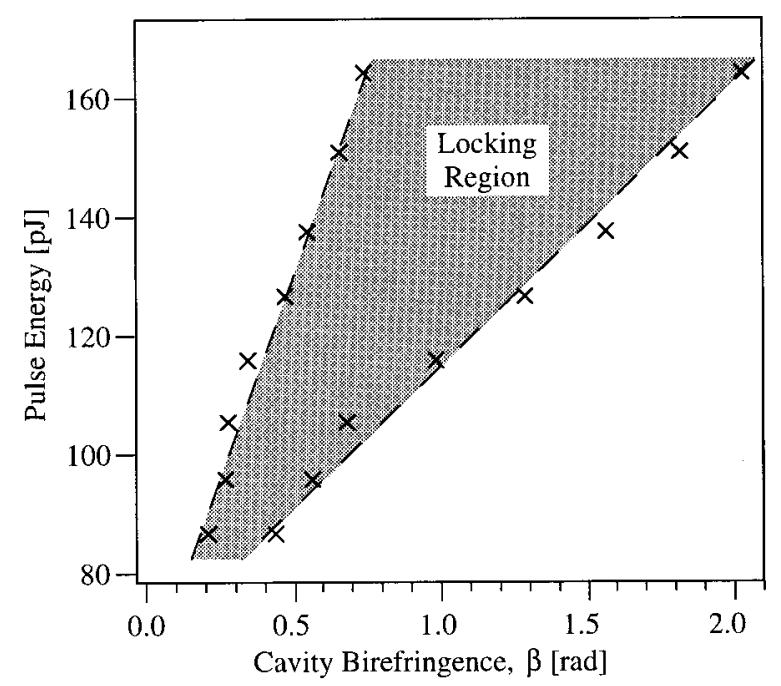

Fig. 11. Plot of the linearly polarized locking region versus intracavity pulse energy and round-trip birefringence, $\beta$.

fringences. For the case of a large pulse energy and/or small linear birefringence the nonlinear birefringence is larger than the linear birefringence. Since the nonlinear birefringence is polarization-state dependent, it can significantly enhance or overcompensate the linear birefringence depending on its local polarization state. Thus the evolution of the polarization is due to both the linear and the nonlinear birefringence with neither axis being stable. In this regime the PEF depends significantly on pulse energy, since both the linear and the nonlinear bi- refringences are contributing to the evolution of the polarization state. If the birefringence is increased or the pulse energy is decreased, so that the linear birefringence is significantly larger, the nonlinear birefringence is not large enough to significantly increase the total birefringence when the polarization is aligned with the slow axis. Hence the slow axis is no longer stable. Since the nonlinear birefringence is related to the pulse energy through the peak power, the magnitudes of linear birefringence where the stability transitions occur are naturally pulse energy dependent, specifically, decreasing with pulse energy. This dependence is confirmed in Fig. 11.

Linearly polarized locking also occurs where the net cavity birefringence is greater than $\pi$ but less than $2 \pi$; however, in these regions the linearly polarized output is aligned along the fast axis. We conjecture that since the nonlinear effects of the pulse can respond only to the average birefringence of the cavity, a fast axis with a birefringence of $2 \pi-\epsilon$ (where $\pi \ll \epsilon<2 \pi$ ), can appear to the nonlinear pulse as an effective slow axis with a birefringence magnitude of $\epsilon$. Thus the relative phase between components can slip by $2 \pi$ each round trip. To achieve $\sim 2 \pi$ of average cavity birefringence within the cavity, the fiber polarization controller paddles must dominate the total cavity birefringence, since each offers only a retardation of $\pi$ (round trip). Therefore the large birefringence is lumped into approximately half the cavity with the $2 \pi$ phase slip occurring on a short length scale with respect to that of the nonlinearity.

\section{CONCLUSION}

In conclusion, we have experimentally observed polarization-locked temporal vector solitons in an opticalfiber laser. The perturbations of the laser are small and occur over lengths short in comparison with the soliton period such that the pulse samples only the averaged characteristics of the cavity. With these small perturbations the system approaches a conservative system. Measurements reveal a $\pm \pi / 2$ relative phase between the orthogonally polarized components of the pulse. The intensity difference, pulse energy dependence, and difference of spectral widths of the experimental pulses agree with theoretical predictions for the PLVS. The interaction of the SPM, XPM, and CEC provides a stabilizing mechanism supplying phase-sensitive negative feedback for perturbation correction. In addition, the instability of the fast axis is clearly observed as the locking of the polarization along the slow axis.

\section{APPENDIX A: NULLING THE BIREFRINGENCE BETWEEN THE OUTPUT COUPLER AND POLARIZATION- STATE ANALYZER/POLARIZER}

To null or compensate all residual birefringence between the output coupler and the polarization-state analyzer and the linear (primary) polarizer (see Fig. 1), all fiber is fastened to the optical table to prevent any movement and change of its birefringence. Also, a bulkhead-style fer- 
rule connector fastened to the optical table is used to couple the connector coated with the output coupler with the uncoated connector. This coupling assembly allows the output coupling connector to be removed without disturbing the position and the birefringence of any of the fiber between the output coupler, polarization-state analyzer, and polarizer. The free-space output from a second laser source is passed through a second linear polarizer (launch polarizer) and coupled into the uncoated connector. Fiber polarization controller \#1 is adjusted until the polarization state at the polarization-state analyzer corresponds to that created by the launch polarizer. The launch polarizer is rotated and fiber polarization controller \#1 adjusted in an iterative manner until the polarization state at the polarization-state analyzer corresponds to that at the launch polarizer in both linearity and azimuthal angle for all angles of the launch polarizer. We adjust fiber polarization controller \#2 with the same process, analyzing the polarization state with the primary polarizer.

\section{ACKNOWLEDGMENTS}

We thank H. A. Haus, C. R. Menyuk, H. Winful, M. Segev, and M. Ablowitz for helpful discussion; G. T. Harvey for lending equipment; and N. Bonadeo for critically reading the manuscript. K. Bergman and B. Collings acknowledge support from Office of Naval Research Grant N00014-96-0773 and National Science Foundation Grant ECS-9502491. The research of J. M. Soto-Crespo was supported by Comunidad de Madrid contract 06T/039/96 and by Directión General de Enseñanza Superior contract PB96-0819.

*B. C. Colling's current address is Bell Laboratories, Lucent Technologies, Holmdel, New Jersey 07733. He can be reached by phone, 732-949-0238; fax, 732-9492473; or e-mail, collings@lucent.com.

\section{REFERENCES}

1. G. P. Agrawal, Nonlinear Fiber Optics, 2nd ed. (Academic, San Diego, Calif., 1995).

2. N. N. Akhmediev and A. Ankiewicz, Solitons, Nonlinear Pulses and Beams (Chapman \& Hall, London, 1997).

3. A. Hasegawa and F. Tappert, "Transmission of stationary nonlinear pulses in dispersive dielectric fiber. I. Anomalous dispersion," Appl. Phys. Lett. 23, 142-144 (1973).

4. L. F. Mollenauer, R. H. Stolen, and J. P. Gordon, "Experimental observation of picosecond narrowing and solitons in optical fibers," Phys. Rev. Lett. 45, 1095-1098 (1980).

5. I. P. Kaminow, "Polarization in optical fibers," IEEE J. Quantum Electron. QE-17, 15-22 (1981).

6. C. D. Poole, N. S. Bergano, R. E. Wagner, and H. J. Schulte, "Polarization dispersion and principal states in a $147-\mathrm{km}$ undersea lightwave cable," J. Lightwave Technol. 6, 11851190 (1988).

7. C. R. Menyuk, "Nonlinear pulse-propagation in birefringent optical fiber," IEEE J. Quantum Electron. QE-23, 174-176 (1987).

8. C. R. Menyuk, "Stability of soliton in birefringent optical fibers. 1. Equal propagation amplitudes," Opt. Lett. 12, 614-616 (1988).

9. C. R. Menyuk, "Stability of soliton in birefringent optical fi- bers. 2. Arbitrary amplitudes," J. Opt. Soc. Am. B 5, 392-402 (1988).

10. M. N. Islam, C. D. Poole, and J. P. Gordon, "Soliton trapping in birefringent optical fibers," Opt. Lett. 14, 10111013 (1989).

11. D. N. Christodoulides and R. I. Joseph, "Vector solitons in birefringent nonlinear dispersive media," Opt. Lett. 13, 53-55 (1988).

12. S. G. Evangelides, Jr., L. F. Mollenauer, J. P. Gordon, and N. S. Bergano, "Polarization multiplexing with solitons," J. Lightwave Technol. 10, 28-35 (1992).

13. M. V. Tratnik and J. E. Sipe, "Bound solitary waves in a birefringent optical fiber," Phys. Rev. A 38, 2011-2017 (1988).

14. N. Akhmediev, A. Buryak, and J. M. Soto-Crespo, "Elliptically polarized solitons in birefringent optical fibers," Opt. Commun. 112, 278-282 (1994).

15. N. Akhmediev and J. M. Soto-Crespo, "Dynamics of solitonlike pulse propagation in birefringent optical fibers," Phys. Rev. E 49, 5742-5754 (1994).

16. N. N. Akhmediev, A. V. Buryak, J. M. Soto-Crespo, and D. R. Andersen, "Phase-locked stationary soliton states in birefringent nonlinear optical fibers," J. Opt. Soc. Am. B 12, 434-439 (1995).

17. J. M. Soto-Crespo, N. Akhmediev, and A. Ankiewicz, "Stationary solitonlike pulses in biefringent optical fiber," Phys. Rev. E 51, 3547-3555 (1995).

18. Y. Chen and J. Atai, "Femtosecond soliton pulses in birefringent optical fibers," J. Opt. Soc. Am. B 14, 2365-2372 (1997).

19. S. T. Cundiff, B. C. Collings, N. N. Akhmediev, J. M. SotoCrespo, K. Bergman, and W. H. Knox, "Observation of polarization-locked vector solitons in optical fiber," Phys. Rev. Lett. 82, 3988-3991 (1999).

20. J. M. Soto-Crespo, N. N. Akhmediev, B. C. Collings, S. T. Cundiff, K. Bergman, and W. H. Knox, "Polarization-locked temporal vector solitons in a fiber laser: theory," J. Opt. Soc. Am. B 17, 366-372 (2000).

21. B. C. Collings, K. Bergman, S. T. Cundiff, S. Tsuda, J. N Kutz, M. Koch, and W. H. Knox, "Short cavity erbium/ ytterbium fiber lasers mode-locked with a saturable Bragg reflector," IEEE J. Sel. Top. Quantum Electron. 3, 10651075 (1997).

22. H. G. Winful, "Polarization instabilities in birefringent nonlinear media: application to fiber-optic devices," Opt. Lett. 11, 33-35 (1986).

23. K. J. Blow, N. J. Doran, and D. Wood, "Polarization instabilities for solitons in birefringent fibers," Opt. Lett. 12, 202-204 (1987).

24. Y. Barad and Y. Silberberg, "Polarization evolution and polarization instability of solitons in a birefringent optical fiber," Phys. Rev. Lett. 78, 3290-3293 (1997).

25. S. V. Manakov, "On the theory of two-dimensional selffocusing of electromagnetic waves," Sov. Phys. JETP 38, 248-253 (1974).

26. J. U. Kang, G. I. Stegeman, J. S. Aitchison, and N. Akhmediev, "Observation of Manakov spatial solitons in AlGaAs planar waveguides," Phys. Rev. Lett. 76, 3699-3702 (1996).

27. L. F. Mollenauer, K. Smith, J. P. Gordon, and C. R. Menyuk, "Resistance of solitons to the effects of polarization dispersion in optical fibers," Opt. Lett. 14, 1219-1221 (1989).

28. S. Tsuda, W. H. Knox, S. T. Cundiff, W. Y. Jan, and J. E. Cunningham, "Mode-locking ultrafast solid-state lasers with saturable Bragg reflectors," IEEE J. Sel. Top. Quantum Electron. 2, 454-464 (1996).

29. J. N. Kutz, B. C. Collings, K. Bergman, S. Tsuda, S. Cundiff, W. H. Knox, P. Holmes, and M. I. Weinstein, "Modelocking pulse dynamics in a fiber laser with a saturable Bragg reflector," J. Opt. Soc. Am. B 14, 2681-2690 (1997).

30. N. N. Akhmediev, J. M. Soto-Crespo, S. T. Cundiff, B. C. Collings, and W. H. Knox, "Phase locking and periodic evolution of solitons in passively mode-locked fiber lasers with a semiconductor saturable absorber," Opt. Lett. 23, 852854 (1998). 
31. S. M. J. Kelley, "Characteristic side-band instability of periodically amplified average soliton," Electron. Lett. 28, 806-807 (1992).

32. J. P. Gordon, "Dispersive perturbation of solitons of the nonlinear Schrödinger-equation," J. Opt. Soc. Am. B 9, 91-97 (1992).

33. H. C. Lefevre, "Single-mode fiber fractional wave devices and polarization controllers," Electron. Lett. 16, 778-780 (1980).

34. S. T. Cundiff, B. C. Collings, and W. H. Knox, "Polarization locking in an isotropic, mode-locked soliton Er/Yb fiber laser," Opt. Express 1, 12-20 (1997); http://epubs.osa.org/ opticsexpress. 